\title{
Molecular Composition of Vestibular Hair Bundles
}

\author{
Jocelyn F. Krey and Peter G. Barr-Gillespie \\ Oregon Hearing Research Center and Vollum Institute, Oregon Health \& Science University, Portland, \\ Oregon 97239 \\ Correspondence: gillespp@ohsu.edu
}

The vertebrate hair bundle, responsible for transduction of mechanical signals into receptor potentials in sensory hair cells, is an evolutionary masterpiece. Composed of actin-filled stereocilia of precisely regulated length, width, and number, the structure of the hair bundle is optimized for sensing auditory and vestibular stimuli. Recent developments in identifying the lipids and proteins constituting the hair bundle, obtained through genetics, biochemistry, and imaging, now permit a description of the consensus composition of vestibular bundles of mouse, rat, and chick.

$\mathrm{H}$ air bundles of vestibular hair cells transduce movements of the head into stimulus-dependent electrical signals; when propagated to the central nervous system, these signals endow our sense of balance. Vestibular bundles contain 50-100 actin-filled stereocilia, arranged in 10-15 ranks of successively increasing height (Fig. 1A), giving the bundle a staircase-like appearance. Stereocilia are relatively uniform in diameter over most of their length but narrow at their apical insertion into the hair cell's soma (Fig. 1B); this "ankle" allows stereocilia to pivot freely when deflected. Stereocilia are coupled together using several types of linkages, including the tip links and ankle links (Fig. 1B); because of these links, a vestibular bundle moves as a unit when stimulated at a single point. Tip links are arranged along the bundle's axis of mirror symmetry, which corresponds to its physiologically responsive axis; bundle deflections toward the tallest stereocilia tense the tip links and open cation-selective transduction channels, whereas deflections in the opposite direction close channels (Fettiplace and Kim 2014). Perpendicular deflections have no effect. The stereocilia staircase is essential for the ability of bundles to transduce mechanical deflections with a defined polarity. Despite our detailed biophysical understanding of the bundle, only in recent years has its molecular composition begun to come into view.

Knowing the identities and abundances of all the components of an organelle-like the vestibular hair bundle-helps one determine how it operates. Characterization of the bundle's composition has proceeded very slowly, however, because of the small number of hair cells (e.g., only $\sim 3000$ in a mature mouse vestibular organ), inaccessibility of the sensory organs within the skull, challenges in isolating bundles, and the small numbers of some proteins within bundles (e.g., <100 tip links per cell). Because

Editors: Guy P. Richardson and Christine Petit

Additional Perspectives on Function and Dysfunction of the Cochlea available at www.perspectivesinmedicine.org

Copyright (C) 2019 Cold Spring Harbor Laboratory Press; all rights reserved; doi: 10.1101/cshperspect.a033209

Cite this article as Cold Spring Harb Perspect Med 2019;9:a033209 

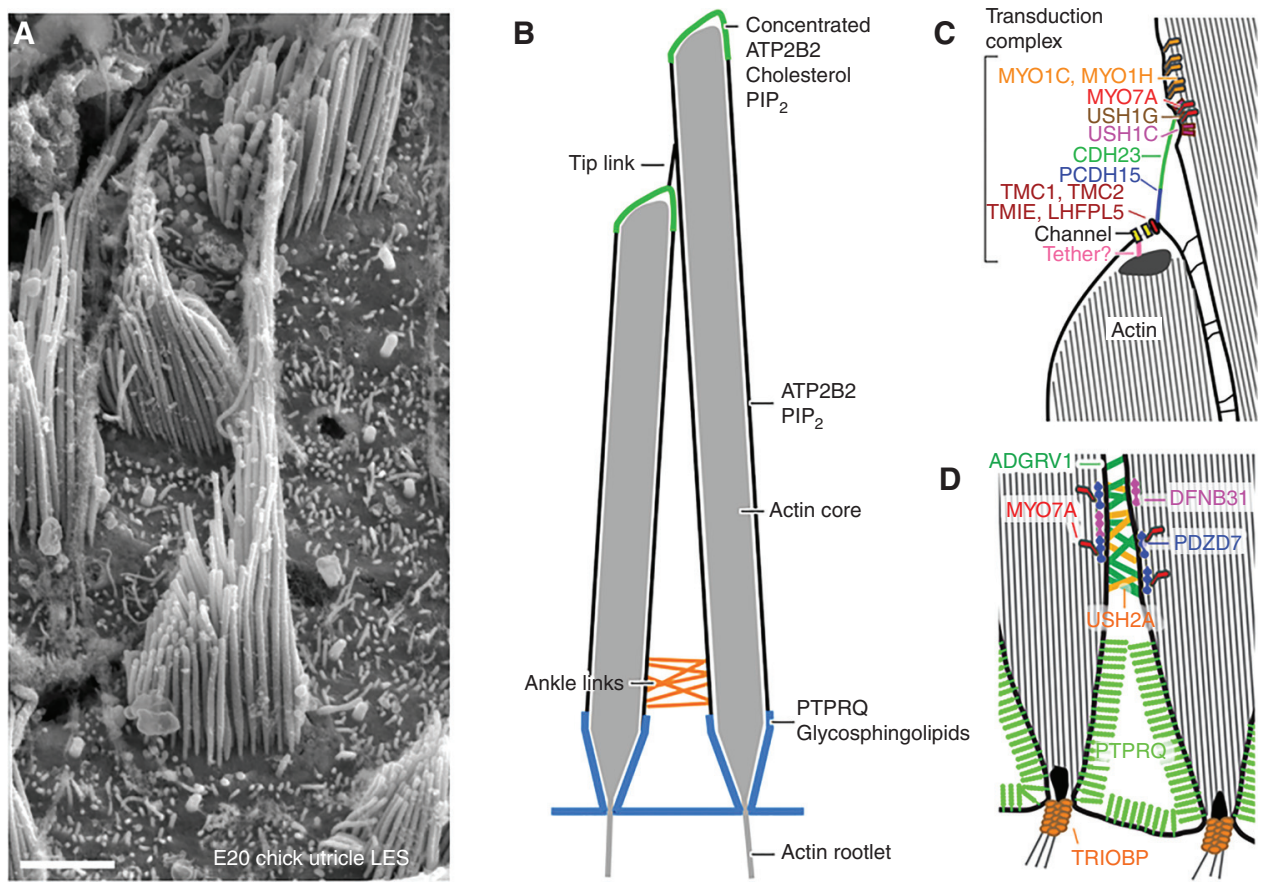

Figure 1. Structure of hair bundles and stereocilia. (A) Hair bundle from the lateral extrastriola (LES) region of a E20 chick utricle. Note staircase appearance of bundle because of systematically increasing height of stereocilia rows. Scale bar, $2 \mu \mathrm{m}$. (B) Major domains of the stereocilia. Tip links, ankle links, and other links bind the stereocilia together. The actin core is a constant cylinder throughout most of its length but is shaped at the tip (beveled toward the tip links) and the bottom (tapered at the insertion into the cell soma). Lipid domains include the glycosphingolipid collar located in the tapers, below the ankle links (PTPRQ [protein tyrosine phosphatase, receptor type Q] partitions there); the shaft domain, with $\mathrm{PIP}_{2}$ and the $\mathrm{Ca}^{2+}$ pump ATP2B2; and the tip domain, with cholesterol and higher levels of $\mathrm{PIP}_{2}$ and ATP2B2. (C) A plausible layout of the transduction complexes at either end of the tip link. The upper complex controls tension on the tip link, and the lower complex includes the transduction channel itself. (D) The ankle link complex. ADGRV1 (adhesion G protein-coupled receptor V1) and USH2A (usherin) are the major link proteins, whereas DFNB31 (whirlin) and PDZD7 (PDZ domain containing 7) anchor those proteins to the cytoskeleton, perhaps via MYO7A (myosin VIIA).

an approach using genetics does not suffer from these problems, identifying "deafness genes" has been the most fruitful way to identify key molecules for bundle function. Unfortunately, defining the bundle's composition using only genetics provides an incomplete view of the bundle's constituents, and yields no information about their relative abundances. Some proteins that control the bundle's structure and function may be inaccessible with an approach using genetics, including those used in essential early developmental steps or those with close paralogs that can compensate for a missing protein. For this reason, we have complemented the genetics approach by determining the complete protein content of hair bundles of chick, mouse, and rat utricle vestibular organs of the inner ear (Shin et al. 2007, 2010, 2013; Krey et al. 2015; Wilmarth et al. 2015). Moreover, because bundles are built with lipids and glycoconjugates as well as proteins, we and others have characterized these molecules as well. Together these data provide a still-developing view of the molecular composition of the vestibular hair bundle.

\section{PROTEINS OF HAIR BUNDLES}

\section{Consensus Hair-Bundle Proteome}

In this review, we describe a consensus vestibular hair-bundle proteome, which includes those 
proteins that are shared between utricular hair bundles of chick, mouse, and rat. This consensus proteome was derived by comparing seven mass spectrometry data sets (see Supplemental Table S1), each of which contains both bundles and whole utricle or utricular epithelium; technical descriptions of the data sets have been published (Krey et al. 2015; Wilmarth et al. 2015). The present cross-species analysis differs from a previous one (Wilmarth et al. 2015) by also including mouse bundles isolated at P3-P7. The individual data sets, including the analysis of each protein's abundance, are available from ProteomeXchange (Vizcaíno et al. 2016) with the following accession numbers: chick Orbitrap, PXD002445; chick LTQ, PXD002410; chick Velos, PXD002414; mouse Orbitrap, PXD002167; mouse Velos, PXD002415; and rat LTQ, PXD002416.

We used methods previously described (Wilmarth et al. 2015) to identify orthologs in each of the three species analyzed. Like with most mass spectrometry analyses, we identified and quantified trypsin-digested peptides, which are then assembled together to identify and quantify proteins. This assembly step is confounded by the protein-grouping problem (Nesvizhskii and Aebersold 2005), however, as more than one gene product may share the same peptide. Each of the data sets used here combines proteins sharing peptides into protein groups, and quantitation is performed at the proteingroup level. Because protein grouping varies from data set to data set, for determining the core proteome, we combined all intersecting protein groups into larger final protein groups (Wilmarth et al. 2015). For simplicity, we generally refer to these protein groups as proteins, although their complete identifier composition is recorded in Supplemental Table S2.

We included in the core proteome of vestibular hair bundles those proteins detected in all three chick data sets, each consisting of $\sim 1800$ detected bundle proteins, and at least two of the five rodent data sets, each consisting of fewer than 1000 proteins (see Supplemental Table S2). As so defined, the core bundle proteome comprised 541 proteins; each of these proteins was also detected in whole utricular epithelium samples, allowing us to calculate their bundleto-epithelium enrichment values. Proteins were each assigned to single functional classes, color coded in this review's figures, which were created for analysis of bundle proteins (Shin et al. 2013) and are more useful for this narrow purpose than are gene ontology terms (Ashburner et al. 2000).

We coupled detection with quantitation of each protein in hair bundles and whole epithelium. Although detection of a protein in bundles does not assure that it is functionally relevant there, the proteins that have high bundle-to-epithelium enrichment usually are important for hearing and balance (Shin et al. 2013). Although we used several methods for quantifying proteins in these data sets (Krey et al. 2015; Wilmarth et al. 2015), all of the methods give an approximately linear relationship between the quantification measure and the molar abundance of a protein in the sample (Krey et al. 2014). Nevertheless, the estimated molar abundance may be less accurate for proteins that are 1 part in $10^{5}$ or less of the total (Krey et al. 2014).

The total molar abundance of proteins assigned to each functional class is represented in Figure 2. As expected, hair bundles have a preponderance of proteins associated with the actin cytoskeleton. The second, third, and fourth most abundant classes of proteins are those involved in energy metabolism (Shin et al. 2007); those associated with microtubules, because of the presence of the axonemal kinocilium (Kikuchi et al. 1989); and those involved in ion homeostasis, including proteins that buffer or extrude $\mathrm{Ca}^{2+}$ (Edmonds et al. 2000; Dumont et al. 2001; Heller et al. 2002). The distribution of protein classes differs substantially between the utricular epithelium and bundles, highlighting the unique protein composition of bundles.

Actin itself accounts for $\sim 50 \%$ of the protein molecules in a hair bundle. Because we can independently estimate the number of actin molecules per stereocilium ( 400,000 in chick) using morphology (Shin et al. 2013), we use actin as the "proteomic ruler" (Wiśniewski et al. 2014) to estimate the abundance of all other bundle proteins. Although the number of actin molecules per stereocilium may be somewhat higher 

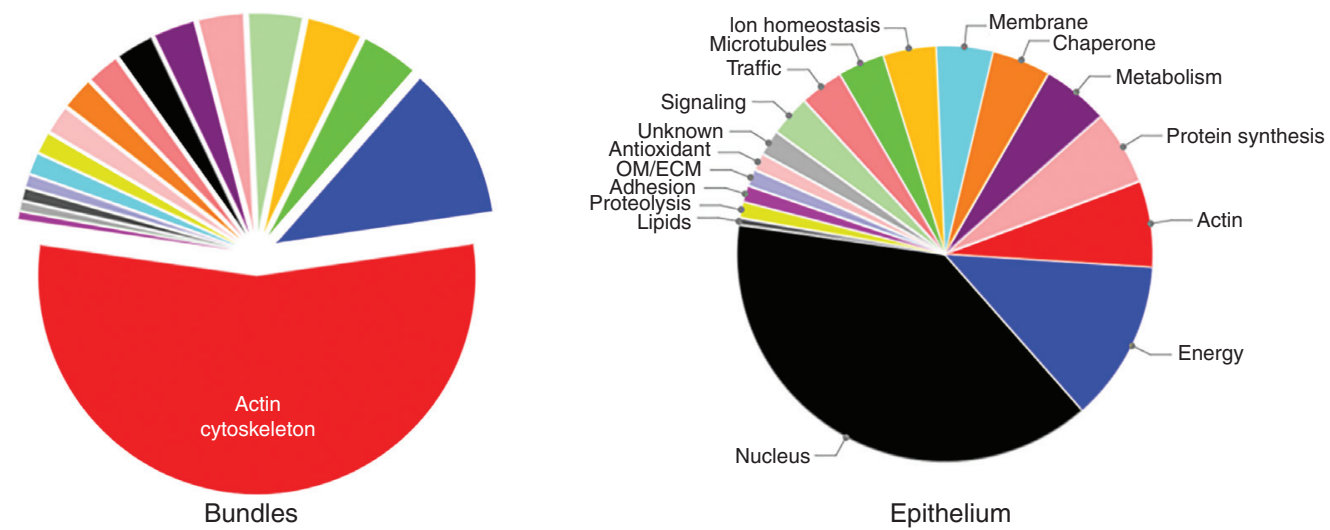

Figure 2. Bundle-epithelium distribution of proteins detected in consensus hair-bundle proteome. In this and following figures, only proteins that were detected in $3 / 3$ chick hair-bundle preparations and $\geq 2 / 5$ rodent bundle preparations (541 total proteins or protein groups) were included in the consensus proteome. Relative molar abundances of all detected proteins were summed separately for bundles and epithelium. "Bundles" includes hair bundles plus $\sim 5 \%$ contamination. "Epithelium" includes the whole utricular epithelium, including bundles, after removal of the otolithic membrane. OM/ECM, otolithic membrane and extracellular matrix.

in rodent utricles (Krey et al. 2016b), for our consensus proteome, we still normalized the molecular abundance of each protein to 400,000 actin monomers. To graphically illustrate the relative abundance of the 163 bundle proteins in the core proteome with bundle-toepithelium enrichment of greater than 0.5 , we plotted one point for each 200 copies in Figure 3.

To highlight the proteins that are most concentrated in hair bundles, we plotted the bundleto-epithelium enrichment as a function of the abundance of each of the 541 core proteins, and labeled the highly enriched or abundant molecules (Fig. 4). Most of these enriched proteins are already known to be important for bundles as they are encoded by deafness genes (see Supplemental Table S2); in addition, several others are annotated in the Mouse Genome Informatics (MGI) database as having an early mortality phenotype (see Supplemental Table S2), which could preclude their identification as deafness genes.

\section{Actin and Actin-Associated Molecules}

Of the $\sim 50$ actin-associated proteins or protein groups, just six-PLS1 (plastin 1), FSCN2 (fascin 2), RDX (radixin), MYO6 (myosin 6), XIRP2 (xin actin-binding repeat-containing protein 2), and CLIC5 (chloride intracellular channel protein 5) - account for $>80 \%$ of all actin-associated molecules remaining after actin. Together, hair bundles have $\sim 40,000$ tight actin cross-linkers per stereocilium; these are predominantly PLS1 and FSCN2, with a modest amount of ESPN (espin) (Krey et al. 2016b). Each stereocilium has 7000 molecules in the ezrin-radixin-moesin (ERM) family, which are mostly RDX (Shin et al. 2007, 2013). RDX and CLIC5 ( 1200 molecules) have been proposed to form a complex linking actin to the membrane (Gagnon et al. 2006; Salles et al. 2014), and XIRP2 ( 3000 molecules) may play a similar role (Francis et al. 2015; Scheffer et al. 2015). SLC9A3R2 (solute carrier family 9 , member A3, regulator 2), a ligand of RDX that couples it to PDZ-binding proteins, is present at $\sim 1200$ copies per stereocilium; it may target various PDZ-binding proteins to stereocilia (Shin et al. 2013). Many other actin-associated proteins were present in the core proteome, including myosins MYO1C, MYO1H, MYO3B, MYO5A, MYO6, MYO7A, MYO15A, and members of the myosin II family; actin-capping molecules CAPZA1, CAPZA2, CAPZB, EPS8L2 (epidermal growth factor receptor kinase substrate 8-like protein), GSN 


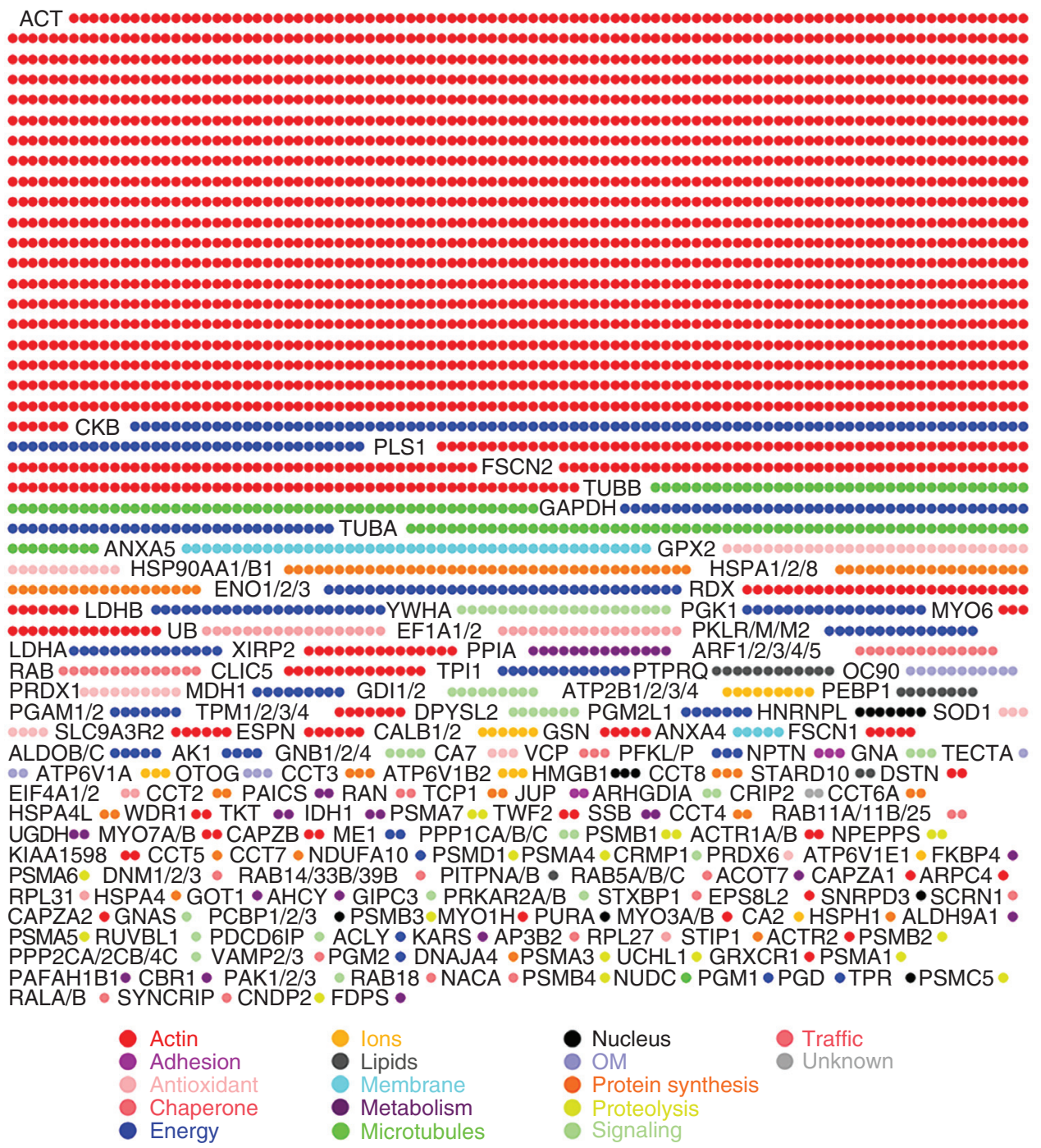

Figure 3. Abundant proteins of consensus hair-bundle proteome. Markers to the right of each protein symbol represent the scaled number of protein molecules per 400,000 molecules of actin; each marker represents 200 protein molecules (rounding up), and there are 100 points per row (excepting labels). When a protein group is plotted, its symbol is a combination of the constituent proteins' symbols (e.g., CALB1/2 for the calbindin; calretinin [CALB1; CALB2] protein group). These abbreviated protein-group symbols are listed in Supplemental Table S2. Only proteins with an enrichment of $>0.5$ and present at $>100$ copies are plotted. From the 163 proteins or protein groups passing these criteria, 667,929 total protein molecules are represented using 3340 markers.

(gelsolin), and TWF2 (twinfilin 2); and actinsevering proteins, such as DSTN (destrin, also known as actin-depolymerizing factor $[\mathrm{ADF}])$. Differences between actin-associated proteins of chick and mouse bundles are relatively modest, although mice stereocilia predominantly use
PLS1 as their actin cross-linker and chick stereocilia use FSCN2 (Fig. 5). MYO6, SLC9A3R2, and FSCN1 are all more abundant in chick stereocilia, whereas the tropomyosins (TPM1-4) and GSN are more abundant in mouse stereocilia (Fig. 5). 
J.F. Krey and P.G. Barr-Gillespie

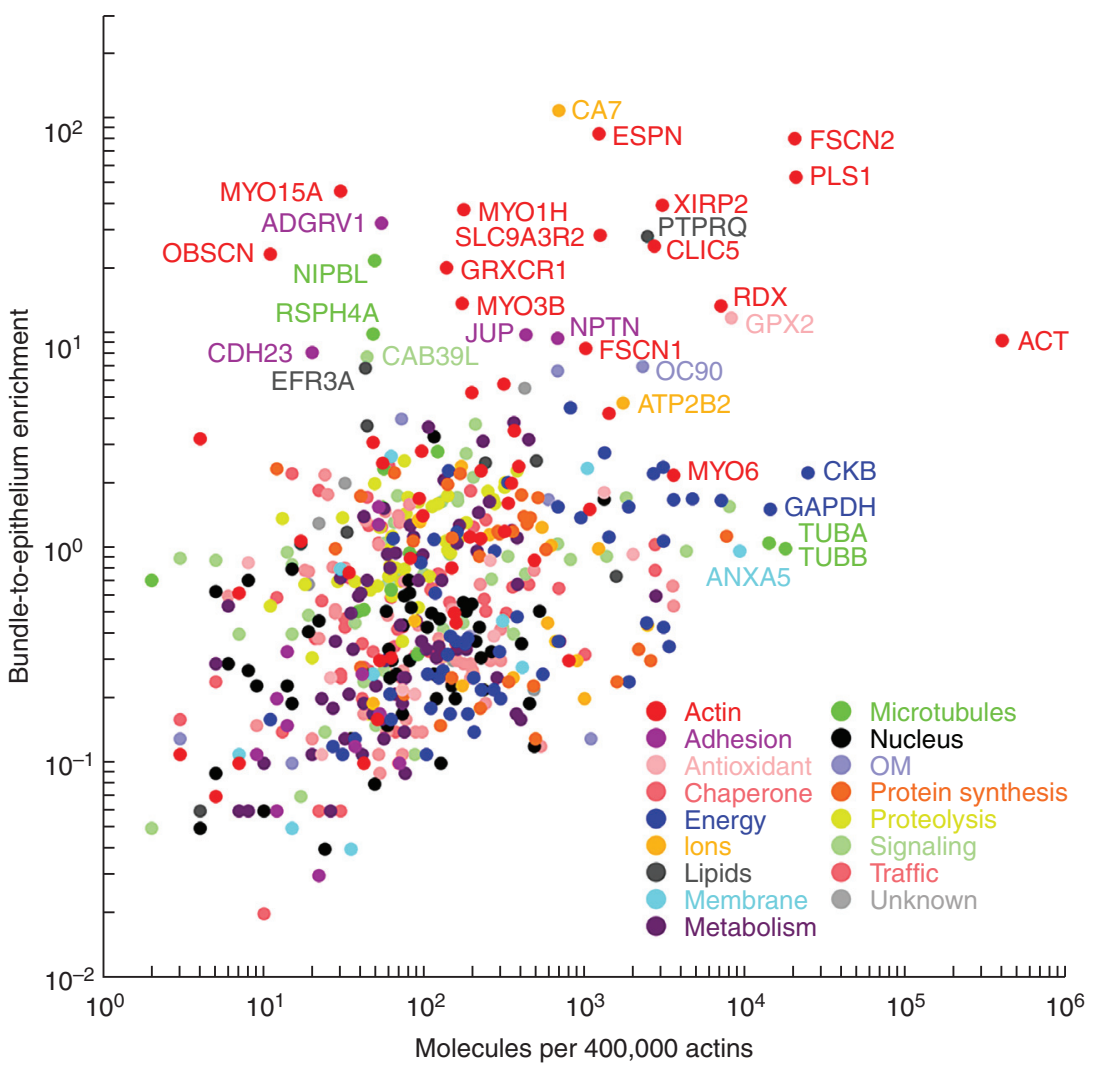

Figure 4. Highly enriched proteins of consensus vestibular hair-bundle proteome. All 541 proteins of the consensus bundle proteome are plotted; selected highly enriched or abundant proteins are labeled. Protein markers and labels are color coded using the classes indicated in the key.
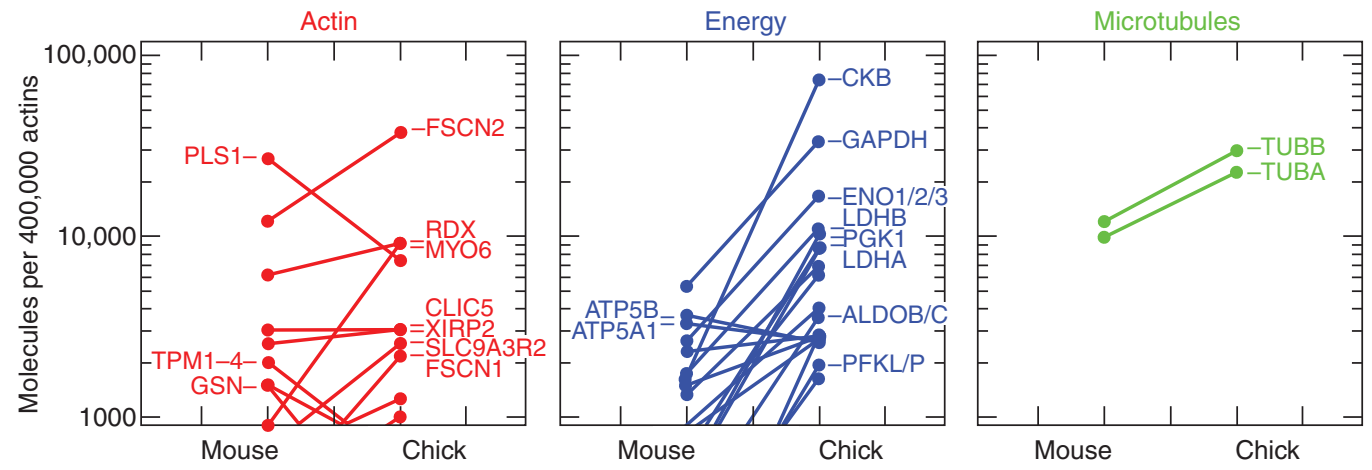

Figure 5. Differences in expression between chick and mouse hair-bundle proteins. Three protein classes are displayed. Many actin-associated proteins are at similar levels in chick and mouse, or the sum of several carrying out the same function. For example, the sum of actin cross-linkers PLS1 (plastin 1) and FSCN2 (fascin 2) is similar in chick and mouse. In contrast, energy metabolism proteins, in particular those involved in glycolysis, are at much higher levels in chick bundles than in mouse bundles. Expression of the tubulin protein groups TUBA and TUBB is about twofold higher in chick, although both remain in approximate stoichiometric equivalence. 
Molecular Composition of Vestibular Hair Bundles

\section{Adhesion}

The most abundant adhesion molecule is NPTN (neuroplastin); its shorter $(55 \mathrm{kDa})$ isoform is expressed in vestibular hair cells and in cochlear hair cells, with expression in outer hair cells being much higher than in inner hair cells (Zeng et al. 2016). NPTN has been recently reported to be associated with ATP2B2 (adenosine triphosphatase $\mathrm{Ca}^{2+}$ transporting plasma membrane 2) (Herrera-Molina et al. 2017; Korthals et al. 2017; Schmidt et al. 2017), and the distribution of NPTN coincides with the distribution of ATP2B2 in hair cells (Dumont et al. 2001). At 700 molecules per stereocilium, NPTN is similar in abundance to the protein group containing ATP2B2 (1700 molecules), consistent with an ATP2B2-anchoring role for NPTN. Ankle and tip-link components ADGRV1 (adhesion G protein-coupled receptor V1, also known as GPR98 [G-protein-coupled receptor 98] or VLGR1 [very large G-protein-coupled receptor 1]) and $\mathrm{CDH} 23$ (cadherin 23) were consistently detected, albeit at $<100$ molecules per stereocilium. Other well-known adhesion molecules, such as PCDH15 (protocadherin 15) and STRC (stereocilin), were detected less consistently, but at levels consistent with their proposed roles. Note that an STRC gene is not annotated in the chicken genome.

\section{Antioxidants}

GPX2 (glutathione peroxidase 2), which protects cells against oxidative damage, is present at high levels in vestibular hair bundles (Fig. 3). Although Gpx2-null mice have no detectable phenotype (Esworthy et al. 2000), other GPX family members could compensate; indeed, both GPX1 and GPX4 were detected in mouse utricular epithelium, although not in bundles (Krey et al. 2016b). Other relatively abundant antioxidants included PRDX1 (peroxiredoxin 1) and SOD1 (superoxide dismutase 1). Oxidative damage plays a major role in noise-induced hearing loss (Henderson et al. 2006), and antioxidants such as GPX2 presumably limit the impact of reactive oxygen species under normal conditions.

\section{Chaperones}

Two chaperone protein groups, corresponding to the HSP90 and HSP70 heat shock protein families (Saibil 2013), are abundant in hair bundles $(\sim 8000$ molecules per stereocilium each). Both protein groups are present at slightly higher levels in bundles than in the whole epithelium, suggesting that they have free diffusional access to stereocilia.

\section{Energy Metabolism}

Proteins involved in glycolysis (e.g., GAPDH [glyceraldehyde 3-phosphate dehydrogenase]) and shuttling of ATP (adenosine triphosphate) (e.g., CKB [creatine kinase B]) were abundant in the core hair-bundle proteome. Although chick hair bundles have high levels of proteins necessary for energy metabolism (Shin et al. 2007), mouse hair bundles have far lower levels of most of these proteins (Fig. 5). Notably, most proteins that were highly enriched in chick bundles over mouse bundles were $\mathrm{CKB}$ and glycolytic enzymes (e.g., ALDOB/C [aldolase B/C], 47-fold; CKB, 45-fold; PGK1 [phosphoglycerate kinase 1], 27-fold; PFKL/P [phosphofructokinase, liver/platelet], 22-fold; LDHA [lactate dehydrogenase A], 18-fold); most other energy proteins, including contaminating mitochondrial proteins, were at similar levels in chick and mouse bundles. Strikingly, this difference does not extend to the whole epithelium; the corresponding chick-to-mouse ratios in the epithelium were ALDOB/C, 4-fold; CKB, 9fold; PGK1, 1.7-fold; PFKL/P, 1.6-fold; and LDHA, 2.4-fold. Thus, whereas glycolytic enzymes and CKB were somewhat more elevated in chick utricular epithelium as compared with mouse, they were much more concentrated in chick stereocilia. Indeed, the average bundle-toepithelium enrichment of these five proteins was 2.2-fold in chick but only 0.7-fold in mouse. Glycolytic enzymes are well known to have significant actin-binding properties (Arnold et al. 1971; Bronstein and Knull 1981; Mejean et al. 1989); perhaps the chicken glycolytic enzymes bind tighter to actin than do their mouse counterparts. 


\section{Ion Homeostasis}

Regulation of $\mathrm{Ca}^{2+}$ is a key function in stereocilia. The protein group containing the mobile $\mathrm{Ca}^{2+}$-binding buffers CALB1 (calbindin) and CALB2 (calretinin) was present in all hair bundles; CALB2 is much more abundant than CALB1 in vestibular hair cells (Edmonds et al. 2000; Spinelli et al. 2012). As already discussed, the $\mathrm{Ca}^{2+}$ pump ATP2B2 was also prominent in the vestibular bundle proteome. Several subunits of the $\mathrm{H}^{+}$-pumping vacuolar ATPase (V-ATPase) were detected at equal levels in bundles and epithelium suggesting a significant presence in stereocilia. The V-ATPase may complement SLC9A9 (Hill et al. 2006) to extrude $\mathrm{H}^{+}$ that enters when $\mathrm{Ca}^{2+}$ is pumped out. Carbonic anhydrases CA2 and CA7 were also present, with CA7 being highly enriched; interestingly, CA7 has been reported to protect cells from oxidative damage (Del Giudice et al. 2013).

\section{Lipid Synthesis and Transport}

Although PEBP1 (phosphatidylethanolaminebinding protein 1) binds phosphatidylethanolamine, phosphatidylinositol, and phosphatidylcholine, this protein is also known as rapidly accelerated fibrosarcoma (RAF) kinase inhibitory protein and regulates several signaling pathways, including the mitogen-activated protein (MAP) kinase pathway, in which it serves to control proliferation and differentiation (Rajkumar et al. 2016). Phosphatidylinositol regulation is pronounced in stereocilia, in keeping with their striking $\mathrm{PIP}_{2}$ membrane domain (Hirono et al. 2004; Zhao et al. 2012). The $\mathrm{PIP}_{2}$ phosphatase PTPRQ rivals ATP2B2 as the most abundant membrane protein present, and the $\mathrm{PIP}_{2}$ synthetic enzymes PI4KA (phosphatidylinositol 4-kinase $\alpha$ ) and PIP4K2A/B (phosphatidylinositol-5-phosphate 4-kinase type-2 $\alpha / \beta$ ) are present in the core proteome (Zhao et al. 2012). A variety of lipid transport proteins are present, including STARD10 (steroidogenic acute regulatory protein-related lipid transfer protein 10), PITPNA (phosphatidylinositol transfer protein $\alpha$ ), PITPNB, OSBP (oxysterolbinding protein 1), and OSBP2. Stereocilia thus actively control their lipid composition, which influences their function (Hirono et al. 2004; Zhao et al. 2012; Peng et al. 2016; Effertz et al. 2017).

\section{Membrane-Associated Proteins}

The most abundant membrane-associated proteins are the annexins, especially ANXA5. Despite being present at 10,000 copies per stereocilium, the functional role of ANXA5 in hair bundles-if any-is not known (Krey et al. 2016a). Several other annexins were also detected, but none were up-regulated in the Anxa5null mouse (Krey et al. 2016a). The transporter ABCA5 (ATP-binding cassette subfamily A member 5) was also detected; it was enriched approximately threefold in bundles.

\section{Metabolism}

The most abundant protein assigned to this class was PPIB (peptidylprolyl isomerase B), which catalyzes cis-trans isomerization of proline imidic peptide bonds in oligopeptides and has been associated with the secretory pathway. Only a few of the proteins in this class were enriched in stereocilia. The exceptions include SSB (Sjogren syndrome antigen B), which is involved in RNA metabolism); FDPS (farnesyl diphosphate synthase), which produces the precursor for lipid modification of some proteins; PAICS (phosphoribosylaminoimidazole carboxylase and phosphoribosylaminoimidazole-succinocarboxamide synthase), which is a bifunctional enzyme essential for purine synthesis; and ACOT7 (acyl coenzyme A (acyl-CoA) thioesterase 7), which produces free fatty acids and acyl-CoA). The role of these or other proteins in this class in hair bundles is unknown.

\section{Microtubules and Axonemes}

Each vestibular hair bundle has a single kinocilium, an axonemal structure made of tubulin. Relative to actin, the TUBA and TUBB protein groups are approximately twofold more abundant in chick stereocilia than in mouse stereocilia (Fig. 5). In frog saccule, the kinocilium has 
the motile $9+2$ microtubule doublet structure (Jacobs and Hudspeth 1990), but in guinea pig utricle, the central pair is missing and inner dynein arms are absent (Kikuchi et al. 1989). Nevertheless, radial spoke head proteins like RSPH4A are detected by mass spectrometry in both chick and rodent bundles; these proteins form a scaffold for signal transduction affecting the central pair of microtubules and dynein arms. As expected, tubulin $\alpha$ and $\beta$ subunits are relatively abundant in bundles, as are several known axonemal proteins, including dynein subunits DYNC2H1 (used for intraflagellar transport), DYNC1LI1, and DYNC1LI2; kinesins and kinesin light chains KIF5B and $\mathrm{KLC1/2/4}$; as well as NIPBL (nipped-B-like protein), NUDC (nuclear distribution protein $C$ ), DCTN1 (dynactin subunit 1), and DCTN2. Cytoplasmic dynein's heavy chain DYNC1H1 was also detected.

\section{Otolithic Membrane and Extracellular Matrix}

The otolithic membrane couples all of the hair bundles in a vestibular organ to the physiological stimulus, for example the inertial mass of otoconia in the utricle (Lundberg et al. 2015). Well-characterized members of this class identified in bundles include components of the otolithic membrane, such as TECTA ( $\alpha$-tectorin), OTOG (otogelin), OTGL (otogelin-like protein), OTOA (otoancorin), as well as otoconia proteins OC90 (otoconin 90) and OTOL1 (otolin 1 ). We detected the tectorial membrane component TECTB in chick bundles and epithelium, but not in mice; TECTB is smaller, however, and may not be efficiently detected by mass spectrometry. We detected two collagens (COL14A1, COL18A1); neither is detected in cochlear tectorial membranes ( $G$ Richardson and P BarrGillespie, unpubl.) but could be within the extracellular matrix surrounding the bundle (Jacobs and Hudspeth 1990).

\section{Protein Synthesis}

In some peripheral domains of cells, for example the leading edge of motile cells, growth cones, and dendrites, local protein translation can sup- port specific functional requirements. In addition to the translation initiation and elongation factors EIF4A1/2, EF1A1/2, EEF2, many ribosome subunits were detected in hair bundles, as were several tRNA-synthetases. Although some ribosome subunits may not be readily detected by mass spectrometry, that most were detected suggests that ribosomes are present in bundles. If so, the absence of rough endoplasmic reticulum in bundles indicates that they would only be used for translation of cytoplasmic proteins, including cytoskeletal proteins like actin (Eom et al. 2003). These are also abundant in the whole epithelium, however, and the ribosome concentration in bundles is lower than that in cell somas.

\section{Proteolysis}

Proteins involved in ubiquitin-mediated proteolysis were abundant in hair bundles, including ubiquitin itself (UB, which is expressed from the Ubb, Ubc, Ubd, and Rps27a genes); components of the SCF (Skp, Cullin, F-box) E3 ubiquitin protein ligase complex, including $\mathrm{FBXO} 2$ (F-box only protein 2) and SKP1A (S-phase kinase-associated protein 1); and the ubiquitin activator UBA1. Polyubiquitin-marked proteins are targeted to the proteasome, and many proteasome subunits were identified in hair bundles. The role of proteolysis in bundle function is not known.

\section{Signaling}

The guanine-nucleotide dissociation inhibitors GDI1 and GDI2, which serve to localize Ras homolog (RHO) family members to membranes, were present at a total of $\sim 2000$ copies per stereocilium. RHO family members themselves were less abundant-RHOA/C, 309 copies per stereocilium; CDC42 (cell division control protein 42 homolog)/RHOG, 170 copies; and RAC1/3 (Ras-related C3 botulinum toxin substrate 1/3), 92 copies. The RHO family has long been predicted to participate in formation of the hair bundle (Kollmar 1999), but how RHO family members are activated and the identity of their downstream targets within the bundle are still unknown. Heterotrimeric G pro- 
teins were also abundant; in addition to 810 copies per stereocilium of $\beta$ subunits, there were 900 copies of $\alpha$ subunits in three separate protein groups. ADGRV1 is the only known Gprotein-coupled receptor in stereocilia, although a role in signaling has not been established.

\section{Trafficking}

Although many proteins associated with intracellular trafficking were detected reproducibly in hair bundles, relatively few of them had high bundle-to-epithelium enrichment. Major trafficking families represented in bundles included the RABs (four large protein groups were detected) and the ARFs (adenosine diphosphate ribosylation factors); peptides present in ARFs 1-5 were all detected at least once, as were those in ARF6. Other prominent trafficking families included clathrin (CLTC, CLTC1), dynamin (DNM1-3), and the adapter protein complex family (including AP1B1, AP2B1, AP2A1, and AP2A2). Turnover of the apical membrane of the hair cell has been reported to be fast (Griesinger et al. 2002), suggesting robust trafficking; evidence for this can be seen in the large number of coated and uncoated vesicles in the pericuticular necklace (Kachar et al. 1997).

\section{PROTEIN COMPLEXES}

The transduction complex (Fig. 1C) contains PCDH15 and CDH23, the cadherins making up the tip link, as well as protein complexes at either end of the tip link. At the shorter stereocilium, four transmembrane proteins, TMC1, TMC2, TMIE, and LHFPL5 (lipoma high-mobility group isoform $\mathrm{C}$ fusion partner-like 5), are all essential for normal transduction (Zhao and Müller 2015). The TMC molecules have been proposed to be the pore-forming components of the transduction channel, although final proof is still elusive (Corey and Holt 2016; Wu and Müller 2016). At the $\mathrm{CDH} 23$ end of the tip link, several myosin motor proteins are present (MYO7A, MYO1C, MYO1H); one or more of these motors may control the resting tension on the transduction channel and mediate the slow form of adaptation. MYO1C can bind di- rectly to $\mathrm{CDH} 23$ (Siemens et al. 2004), whereas MYO7A couples to CDH23 through USH1C (harmonin) and USH1G (sans) (Pan and Zhang 2012).

Unfortunately, transduction-complex proteins have been difficult to detect in mass spectrometry analysis of whole hair bundles, presumably for three principle reasons. First, the proteins are at very low abundance; a rough estimate of concentration indicates that only 1$10 \mathrm{amol}$ of protein is present per chick or mouse utricle's worth of hair bundles. Second, although mass spectrometry is sufficient to detect low amounts of protein if sufficient numbers of utricles are used to prepare samples, the transduction proteins are estimated to be present at less than 1 part in 100,000 in bundles. Unlike many next-generation DNA-sequencing technologies, mass spectrometry of peptides has a limited dynamic range, so detection of such rare proteins is difficult when so many other proteins are present. Finally, many of the key transduction proteins contain multiple transmembrane domains. Peptides derived from transmembrane domains are hydrophobic and behave poorly in mass spectrometry, so the relative detectability of transmembrane domain-containing proteins is less than hydrophilic proteins. The tip-link proteins $\mathrm{PCDH} 15$ and $\mathrm{CDH} 23$ are routinely detected by mass spectrometry, but they are very large (so there are many more peptides per mole of protein, increasing the probability of detection), they each have only a single transmembrane domain (minimizing the number of hydrophobic peptides), and they form developmental and kinocilial links (increasing their abundance). Likewise, the motors MYO7A, MYO1C, and $\mathrm{MYO} 1 \mathrm{H}$ are routinely detected, but they are present at substantially more than one copy per transduction complex.

The composition of the ankle-link complex has also begun to emerge (Fig. 1D). Two proteins with very large extracellular domains, ADGRV1 and USH2A, make up the ankle links themselves (Adato et al. 2005; McGee et al. 2006). Through direct or indirect interactions, MYO7A localizes some of the ankle-link components within stereocilia, including ADGRV1 and the PDZ-domain proteins DFNB31 and 
PDZD7 (Michalski et al. 2007; Morgan et al. 2016; Zou et al. 2017).

Accurate estimates of the abundance of large protein complexes with known subunit stoichiometry can be made from mass spectrometry data; protein-to-protein variability can be overcome by averaging across subunits. The proteasome is a good example; its core $20 \mathrm{~S}$ particle is made from rings of seven $\alpha$ (PSMA1-7) and $\beta$ subunits (PSMB1-7), with several additional proteins used in tissue-specific proteasomes (Murata et al. 2009). The average molar abundance of the detected subunits suggests that there are $200 \pm 90$ proteasomes per stereocilium; their bundle-to-epithelium enrichment of $1.5 \pm$ 0.5 suggests that they are slightly concentrated in stereocilia, reinforcing the importance of proteolysis for stereocilia function.

Another example is the ribosome, which is composed of $\sim 80$ protein subunits, most of which are present in single copies (Westermann et al. 1976; Anger et al. 2013; Slavov et al. 2015). We reproducibly detected 27 ribosome subunits in the chick, and their average molar abundance suggests that there are $150 \pm 80$ ribosomes per stereocilium. With an enrichment of $0.3 \pm 0.1$, ribosomes apparently are largely excluded from stereocilia; however, local protein synthesis may be present but not prominent in vestibular hair bundles.

\section{LIPIDS OF HAIR BUNDLES}

Often overlooked because of the relatively few tools for characterization, the lipid components of the hair bundle are also vitally important. The membrane of a bundle is continuous with the apical membrane of the hair cell and fits over the actin-rich stereocilia like a glove on a finger. Locating the bundle on the apical membrane allows the cell to use domain-targeting mechanisms that allow for a very different lipid and protein composition from that of the basolateral membrane. Moreover, the bundle establishes lipid domains that are distinct from those of the apical surface, despite the continuity of the membrane.

At least 182 lipid species are present in chick hair bundles, with $96 \%$ of the lipid mass deriving from only 76 species (Zhao et al. 2012). Al-
Molecular Composition of Vestibular Hair Bundles

though the head-group distribution is not unusual and is similar in bundles and epithelium, bundles are distinguished by the composition of their sphingomyelin and ceramide acyl chains, which are nearly exclusively short and saturated (i.e., 16:0). Acyl chains like these are typically found in relatively gel-like domains (Zhao et al. 2012).

The hair bundle is particularly notable for its large-scale spatial segregation of lipids. Glycosphingolipids, including those detected by cholera toxin B (CTB), are found at the stereocilia taper region; the lipid phosphatase PTPRQ also localizes to this domain (Zhao et al. 2012). GM3 synthase (ST3GAL5), which is essential for forming GM3 ganglioside and all a- and b-series gangliosides (Iber and Sandhoff 1989; Yoshikawa et al. 2015), is required for auditory function (Yoshikawa et al. 2015). The ganglioside domain may form a diffusional barrier for lipids exchanging between stereocilia and the cell's apical surface; likewise, it might also constrain stereocilia proteins. In deaf St3gal5 ${ }^{\mathrm{KO}}$ mice, PTPRQ is distributed throughout the stereocilia (Yoshikawa et al. 2015); one interpretation of this result is that the ganglioside domain constrains PTPRQ to stereocilia bases, and so loss of gangliosides releases PTPRQ. PTPRQ is not basally restricted in all hair cells; however, in extrastriolar hair cells of mouse and chick utricles, PTPRQ is spread throughout the hair bundle. Whether there is a basal ganglioside domain in these cells has not yet been examined.

Compared to the gangliosides, $\mathrm{PIP}_{2}$ has a reciprocal distribution in stereocilia, as it is missing from the tapers but present in the stereocilia shafts and tips; this domain is also marked by presence of the calcium pump ATP2B2 (Zhao et al. 2012). The boundary between these two lipid domains appears to control activation of the actin-to-membrane linker RDX, which is phosphorylated at this boundary (Zhao et al. 2012). The ganglioside domain may also prevent diffusion of $\mathrm{PIP}_{2}$ out of stereocilia, compartmentalizing signaling with this lipid.

\section{GLYCOCALYX OF HAIR BUNDLES}

Glycoconjugates of proteins and lipids are also more difficult to characterize than are proteins 
and thus are poorly understood in hair bundles. As assayed by lectins and polycation probes, stereocilia have a robust glycocalyx covering their surface (Flock et al. 1977; Santi and Anderson, 1987; Takumida et al. 1988; Goodyear and Richardson 1994). The glycocalyx is apparently needed for an essential lubrication task-stereocilia must contact each other yet slide readily and not fuse. Counterions like $\mathrm{Ca}^{2+}$ bridge the negatively charged glycocalyx of each stereocilium, providing cohesion and permitting sliding (LeBoeuf et al. 2011).

Whereas the ganglioside lipid domain at the stereocilia base is part of the glycocalyx, glycoproteins likely also contribute significantly to the glycocalyx in the rest of the stereocilia (Plinkert et al. 1992). Indeed, neuraminidasesensitive reactivity of the tip link with cationic ferritin and colloidal thorium suggest that it too is modified with carbohydrate chains, including those with sialic acid (van Benthem et al. 1993).

Application of glycomics methods-mass spectrometry of carbohydrate conjugates of proteins and lipids-could be valuable for better understanding the hair bundle's glycocalyx. Because of their heterogeneity, lability of modifications, and minimal differences in structure, as well as the lack of a comprehensive database of biologically possible structures, carbohydrate chains are inherently more difficult to analyze than are proteins. Modern mass spectrometers and improved analysis techniques have led to a substantially improved ability to thoroughly characterize glycoconjugates, however (Kailemia et al. 2014).

\section{CONCLUDING REMARKS}

Although the hair bundle's protein and lipid content are now relatively well understood, having this parts list is only an initial step in understanding how the bundle is assembled, carries out mechanotransduction, and is regulated (Barr-Gillespie 2015). Although many of the most prominent bundle proteins have been localized, how their locations change as they target to the bundle and during bundle construction is poorly understood. Further insight will require characterization of posttranslational modifications that alter the protein's activity and interactions, as well as knowledge of how each protein interacts with other bundle proteins.

Application of highly selective biochemical techniques, such as hair-bundle purification coupled with efficient immunoaffinity enrichment, allows for determination of stable protein-protein interactions that occur in bundles (Shin et al. 2013; Morgan et al. 2016; Avenarius et al. 2017). Using this approach, we showed that a key cargo of MYO7A in bundles is the PDZ-domain protein PDZD7, and that the capping protein subunit CAPZB interacts with known actin capper TWF2 (Avenarius et al. 2017). Indeed, immunoaffinity enrichment from purified bundles can be used to examine many bundle interactions, even including those among the transduction complex proteins.

Relatively little is known about how auditory and vestibular hair-bundle proteomes differ. A preliminary study noted significant differences when comparing chick cochlea to utricle, but isolation of cochlea hair bundles was very inefficient and only the most abundant proteins were confidently identified (Avenarius et al. 2014). Examination of rodent cochlea bundles will be even more difficult, as the layout of auditory hair cells in a long strip is not conducive to efficient isolation of their bundles. Given the trajectory over the last 25 years, however, future improvement in the detection limit for protein mass spectrometry seems highly likely. Thus, future studies may indeed be able to characterize the proteomes of very small numbers of bundles, including those of cochlear hair cells.

\section{ACKNOWLEDGMENTS}

We thank Rachel Dumont for the scanning electron micrograph of Figure 1A. P.G.B.-G. is supported by National Institutes of Health (NIH) Grants R01 DC002368, R01 DC011034, and P30 DC005983; J.F.K. is supported by NIH Grant R03 DC014544.

\section{REFERENCES}

Adato A, Lefevre G, Delprat B, Michel V, Michalski N, Chardenoux S, Weil D, El-Amraoui A, Petit C. 2005. Usherin, 
the defective protein in Usher syndrome type IIA, is likely to be a component of interstereocilia ankle links in the inner ear sensory cells. Hum Mol Genet 14: 3921-3932.

Anger AM, Armache JP, Berninghausen O, Habeck M, Subklewe M, Wilson DN, Beckmann R. 2013. Structures of the human and Drosophila 80S ribosome. Nature 497: $80-85$.

Arnold H, Henning R, Pette D. 1971. Quantitative comparison of the binding of various glycolytic enzymes to Factin and the interaction of aldolase with G-actin. Eur J Biochem 22: 121-126.

Ashburner M, Ball CA, Blake JA, Botstein D, Butler H, Cherry JM, Davis AP, Dolinski K, Dwight SS, Eppig JT, et al. 2000. Gene ontology: Tool for the unification of biology. The Gene Ontology Consortium. Nat Genet 25: 25-29.

Avenarius MR, Saylor KW, Lundeberg MR, Wilmarth PA, Shin JB, Spinelli KJ, Pagana JM, Andrade L, Kachar B, Choi D, et al. 2014. Correlation of actin crosslinker and capper expression levels with stereocilia growth phases. Mol Cell Proteomics 13: 606-620.

Avenarius MR, Krey JF, Dumont RA, Morgan CP, Benson CB, Vijayakumar S, Cunningham CL, Scheffer DI, Corey DP, Müller U, et al. 2017. Heterodimeric capping protein is required for stereocilia length and width regulation. J Cell Biol 216: 3861-3881.

Barr-Gillespie PG. 2015. Assembly of hair bundles, an amazing problem for cell biology. Mol Biol Cell 26: 2727-2732.

Bronstein WW, Knull HR. 1981. Interaction of muscle glycolytic enzymes with thin filament proteins. Can J Biochem 59: 494-499.

Corey DP, Holt JR. 2016. Are TMCs the mechanotransduction channels of vertebrate hair cells? J Neurosci 36: 10921-10926.

Del Giudice R, Monti DM, Truppo E, Arciello A, Supuran CT, De Simone G, Monti SM. 2013. Human carbonic anhydrase VII protects cells from oxidative damage. Biol Chem 394: 1343-1348.

Dumont RA, Lins U, Filoteo AG, Penniston JT, Kachar B Gillespie PG. 2001. Plasma membrane $\mathrm{Ca}^{2+}$-ATPase isoform 2a is the PMCA of hair bundles. JNeurosci 21: 50665078.

Edmonds B, Reyes R, Schwaller B, Roberts WM. 2000. Calretinin modifies presynaptic calcium signaling in frog saccular hair cells. Nat Neurosci 3: 786-790.

Effertz T, Becker L, Peng AW, Ricci AJ. 2017. Phosphoinositol-4,5-bisphosphate regulates auditory hair cell mechanotransduction channel pore properties and fast adaptation. J Neurosci 37: 11632-11646.

Eom T, Antar LN, Singer RH, Bassell GJ. 2003. Localization of a $\beta$-actin messenger ribonucleoprotein complex with zipcode-binding protein modulates the density of dendritic filopodia and filopodial synapses. J Neurosci 23: 10433-10444.

Esworthy RS, Mann JR, Sam M, Chu FF. 2000. Low glutathione peroxidase activity in Gpxl knockout mice protects jejunum crypts from $\gamma$-irradiation damage. Am J Physiol Gastrointest Liver Physiol 279: G426-G436.
Fettiplace R, Kim KX. 2014. The physiology of mechanoelectrical transduction channels in hearing. Physiol Rev 94: 951-986

Flock A, Flock B, Murray E. 1977. Studies on the sensory hairs of receptor cells in the inner ear. Acta Otolaryngol 83: 85-91.

Francis SP, Krey JF, Krystofiak ES, Cui R, Nanda S, Xu W, Kachar B, Barr-Gillespie PG, Shin JB. 2015. A short splice form of Xin-actin binding repeat containing 2 (XIRP2) lacking the Xin repeats is required for maintenance of stereocilia morphology and hearing function. J Neurosci 35: 1999-2014.

Gagnon LH, Longo-Guess CM, Berryman M, Shin JB, Saylor KW, Yu H, Gillespie PG, Johnson KR. 2006. The chloride intracellular channel protein CLIC5 is expressed at high levels in hair cell stereocilia and is essential for normal inner ear function. J Neurosci 26: 10188-10198.

Goodyear R, Richardson G. 1994. Differential glycosylation of auditory and vestibular hair bundle proteins revealed by peanut agglutinin. J Comp Neurol 345: 267-278.

Griesinger CB, Richards CD, Ashmore JF. 2002. Fm1-43 reveals membrane recycling in adult inner hair cells of the mammalian cochlea. J Neurosci 22: 3939-3952.

Heller S, Bell AM, Denis CS, Choe Y, Hudspeth AJ. 2002. Parvalbumin 3 is an abundant $\mathrm{Ca}^{2+}$ buffer in hair cells. J Assoc Res Otolaryngol 3: 488-498.

Henderson D, Bielefeld EC, Harris KC, Hu BH. 2006. The role of oxidative stress in noise-induced hearing loss. Ear Hear 27: 1-19.

Herrera-Molina R, Mlinac-Jerkovic K, Ilic K, Stöber F, Vemula SK, Sandoval M, Milosevic NJ, Simic G, Smalla KH, Goldschmidt J, et al. 2017. Neuroplastin deletion in glutamatergic neurons impairs selective brain functions and calcium regulation: Implication for cognitive deterioration. Sci Rep 7: 7273.

Hill JK, Brett CL, Chyou A, Kallay LM, Sakaguchi M, Rao R, Gillespie PG. 2006. Vestibular hair bundles control pH with $\left(\mathrm{Na}^{+}, \mathrm{K}^{+}\right) / \mathrm{H}^{+}$exchangers NHE6 and NHE9. J Neurosci 26: 9944-9955.

Hirono M, Denis CS, Richardson GP, Gillespie PG. 2004. Hair cells require phosphatidylinositol 4,5-bisphosphate for mechanical transduction and adaptation. Neuron 44: 309-320.

Iber H, Sandhoff K. 1989. Identity of GD1C, GT1a and GQ1b synthase in Golgi vesicles from rat liver. FEBS Lett 254: 124-128.

Jacobs RA, Hudspeth AJ. 1990. Ultrastructural correlates of mechanoelectrical transduction in hair cells of the bullfrog's internal ear. Cold Spring Harb Symp Quant Biol 55: 547-561.

Kachar B, Battaglia A, Fex J. 1997. Compartmentalized vesicular traffic around the hair cell cuticular plate. Hear Res 107: 102-112.

Kailemia MJ, Ruhaak LR, Lebrilla CB, Amster IJ. 2014. Oligosaccharide analysis by mass spectrometry: A review of recent developments. Anal Chem 86: 196-212.

Kikuchi T, Takasaka T, Tonosaki A, Watanabe H. 1989. Fine structure of guinea pig vestibular kinocilium. Acta Otolaryngol 108: 26-30. 
Kollmar R. 1999. Who does the hair cell's 'do? Rho GTPases and hair-bundle morphogenesis. Curr Opin Neurobiol 9: 394-398.

Korthals M, Langnaese K, Smalla KH, Kähne T, HerreraMolina R, Handschuh J, Lehmann AC, Mamula D, Naumann M, Seidenbecher C, et al. 2017. A complex of neuroplastin and plasma membrane $\mathrm{Ca}^{2+}$ ATPase controls T cell activation. Sci Rep 7: 8358 .

Krey JF, Wilmarth PA, Shin JB, Klimek J, Sherman NE, Jeffery ED, Choi D, David LL, Barr-Gillespie PG. 2014 Accurate label-free protein quantitation with high- and low-resolution mass spectrometers. J Proteome Res 13 1034-1044.

Krey JF, Sherman NE, Jeffery ED, Choi D, Barr-Gillespie PG. 2015. The proteome of mouse vestibular hair bundles over development. Sci Data 2: 150047.

Krey JF, Drummond M, Foster S, Porsov E, Vijayakumar S, Choi D, Friderici K, Jones SM, Nuttall AL, Barr-Gillespie PG. 2016a. Annexin A5 is the most abundant membraneassociated protein in stereocilia but is dispensable for hair-bundle development and function. Sci Rep 6: 27221.

Krey JF, Krystofiak ES, Dumont RA, Vijayakumar S, Choi D, Rivero F, Kachar B, Jones SM, Barr-Gillespie PG. 2016b. Plastin 1 widens stereocilia by transforming actin filament packing from hexagonal to liquid. J Cell Biol 215: 467482.

LeBoeuf AC, Ó Maoiléidigh D, Hudspeth AJ. 2011. Divalent counterions tether membrane-bound carbohydrates to promote the cohesion of auditory hair bundles. Biophys J 101: 1316-1325.

Lundberg YW, Xu Y, Thiessen KD, Kramer KL. 2015. Mechanisms of otoconia and otolith development. Dev Dyn 244: 239-253.

McGee J, Goodyear RJ, McMillan DR, Stauffer EA, Holt JR, Locke KG, Birch DG, Legan PK, White PC, Walsh EJ, et al. 2006. The very large G-protein-coupled receptor VLGR1: A component of the ankle link complex required for the normal development of auditory hair bundles. J Neurosci 26: 6543-6553.

Mejean C, Pons F, Benyamin Y, Roustan C. 1989. Antigenic probes locate binding sites for the glycolytic enzymes glyceraldehyde-3-phosphate dehydrogenase, aldolase and phosphofructokinase on the actin monomer in microfilaments. Biochem J 264: 671-677.

Michalski N, Michel V, Bahloul A, Lefevre G, Barral J, Yagi H, Chardenoux S, Weil D, Martin P, Hardelin JP, et al 2007. Molecular characterization of the ankle-link complex in cochlear hair cells and its role in the hair bundle functioning. J Neurosci 27: 6478-6488.

Morgan CP, Krey JF, Grati M, Zhao B, Fallen S, KannanSundhari A, Liu XZ, Choi D, Müller U, Barr-Gillespie PG. 2016. PDZD7-MYO7A complex identified in enriched stereocilia membranes. eLife 5: e18312.

Murata S, Yashiroda H, Tanaka K. 2009. Molecular mechanisms of proteasome assembly. Nat Rev Mol Cell Biol 10: 104-115.

Nesvizhskii AI, Aebersold R. 2005. Interpretation of shotgun proteomic data: The protein inference problem. Mol Cell Proteomics 4: 1419-1440.

Pan L, Zhang M. 2012. Structures of usher syndrome 1 proteins and their complexes. Physiology (Bethesda) 27: 2542 .
Peng AW, Gnanasambandam R, Sachs F, Ricci AJ. 2016. Adaptation independent modulation of auditory hair cell mechanotransduction channel open probability implicates a role for the lipid bilayer. J Neurosci 36: $2945-$ 2956.

Plinkert PK, Plinkert B, Zenner HP. 1992. Carbohydrates in the cell surface of hair cells from the guinea pig cochlea. Eur Arch Otorhinolaryngol 249: 67-73.

Rajkumar K, Nichita A, Anoor PK, Raju S, Singh SS, Burgula S. 2016. Understanding perspectives of signalling mechanisms regulating PEBP1 function. Cell Biochem Funct 34: $394-403$

Saibil H. 2013. Chaperone machines for protein folding, unfolding and disaggregation. Nat Rev Mol Cell Biol 14: 630-642.

Salles FT, Andrade LR, Tanda S, Grati M, Plona KL, Gagnon LH, Johnson KR, Kachar B, Berryman MA. 2014. CLIC5 stabilizes membrane-actin filament linkages at the base of hair cell stereocilia in a molecular complex with radixin, taperin, and myosin VI. Cytoskeleton (Hoboken) 71: 6178.

Santi PA, Anderson CB. 1987. A newly identified surface coat on cochlear hair cells. Hear Res 27: 47-65.

Scheffer DI, Zhang DS, Shen J, Indzhykulian A, Karavitaki KD, Xu YJ, Wang Q, Lin JJ, Chen ZY, Corey DP. 2015. $\mathrm{XIRP} 2$, an actin-binding protein essential for inner ear hair-cell stereocilia. Cell Rep 10: 1811-1818.

Schmidt N, Kollewe A, Constantin CE, Henrich S, RitzauJost A, Bildl W, Saalbach A, Hallermann S, Kulik A, Fakler B, et al. 2017. Neuroplastin and basigin are essential auxiliary subunits of plasma membrane $\mathrm{Ca}^{2+}$-ATPases and key regulators of $\mathrm{Ca}^{2+}$ clearance. Neuron 96: 827838.e9.

Shin JB, Streiiger F, Beynon A, Peters T, Gadzala L, McMillen D, Bystrom C, Van der Zee CE, Wallimann T, Gillespie PG. 2007. Hair bundles are specialized for ATP delivery via creatine kinase. Neuron 53: 371-386.

Shin JB, Longo-Guess CM, Gagnon LH, Saylor KW, Dumont RA, Spinelli KJ, Pagana JM, Wilmarth PA, David LL, Gillespie PG, et al. 2010. The R109H variant of fascin2 , a developmentally regulated actin crosslinker in haircell stereocilia, underlies early-onset hearing loss of DBA/ 2J mice. J Neurosci 30: 9683-9694.

Shin JB, Krey JF, Hassan A, Metlagel Z, Tauscher AN, Pagana JM, Sherman NE, Jeffery ED, Spinelli KJ, Zhao H, et al. 2013. Molecular architecture of the chick vestibular hair bundle. Nat Neurosci 16: 365-374.

Siemens J, Lillo C, Dumont RA, Reynolds A, Williams DS, Gillespie PG, Müller U. 2004. Cadherin 23 is a component of the tip link in hair-cell stereocilia. Nature 428: 950955.

Slavov N, Semrau S, Airold E, Budnik B, van Oudenaarden A. 2015. Differential stoichiometry among core ribosomal proteins. Cell Rep 13: 865-873.

Spinelli KJ, Klimek J, Wilmarth PA, Shin JB, Choi D, David LL, Gillespie PG. 2012. Distinct energy metabolism of auditory and vestibular sensory epithelia revealed by quantitative mass spectrometry using MS2 intensity. Proc Natl Acad Sci 109: E268-E277.

Takumida M, Wersäll J, Bagger-Sjöbäck D. 1988. Stereociliary glycocalyx and interconnections in the guinea pig vestibular organs. Acta Otolaryngol 106: 130-139. 
Van Benthem PP, de Groot JC, Albers FW, Veldman JE, Huizing EH. 1993. Structure and composition of stereocilia cross-links in normal and hydropic cochleas of the guinea pig. Eur Arch Otorhinolaryngol 250: 73-77.

Vizcaíno JA, Csordas A, del-Toro N, Dianes JA, Griss J, Lavidas I, Mayer G, Perez-Riverol Y, Reisinger F, Ternent T, et al. 2016. 2016 update of the PRIDE database and its related tools. Nucleic Acids Res 44: D447-D456.

Westermann P, Heumann W, Bielka H. 1976. On the stoichiometry of proteins in the small ribosomal subunit of hepatoma ascites cells. FEBS Lett 62: 132-135.

Wilmarth PA, Krey JF, Shin JB, Choi D, David LL, BarrGillespie PG. 2015. Hair-bundle proteomes of avian and mammalian inner-ear utricles. Sci Data 2: 150074.

Wiśniewski JR, Hein MY, Cox J, Mann M. 2014. A "proteomic ruler" for protein copy number and concentration estimation without spike-in standards. Mol Cell Proteomics 13: 3497-3506.

Wu Z, Müller U. 2016. Molecular identity of the mechanotransduction channel in hair cells: Not quiet there yet. J Neurosci 36: 10927-10934.
Molecular Composition of Vestibular Hair Bundles

Yoshikawa M, Go S, Suzuki S, Suzuki A, Katori Y, Morlet T, Gottlieb SM, Fujiwara M, Iwasaki K, Strauss KA, et al. 2015. Ganglioside GM3 is essential for the structural integrity and function of cochlear hair cells. Hum Mo Genet 24: 2796-2807.

Zeng WZ, Grillet N, Dewey JB, Trouillet A, Krey JF, BarrGillespie PG, Oghalai JS, Müller U. 2016. Neuroplastin isoform Np55 is expressed in the stereocilia of outer hair cells and required for normal outer hair cell function. J Neurosci 36: 9201-9216.

Zhao B, Müller U. 2015. The elusive mechanotransduction machinery of hair cells. Curr Opin Neurobiol 34: 172-179.

Zhao H, Williams DE, Shin JB, Brugger B, Gillespie PG. 2012. Large membrane domains in hair bundles specify spatially constricted radixin activation. J Neurosci 32 . 4600-4609.

Zou J, Chen Q, Almishaal A, Mathur PD, Zheng T, Tian C, Zheng QY, Yang J. 2017. The roles of USH1 proteins and PDZ domain-containing USH proteins in USH2 complex integrity in cochlear hair cells. Hum Mol Genet 26: 624636. 


\section{$\&_{\mathrm{CSH}}^{\infty} \&$ Cold Spring Harbor

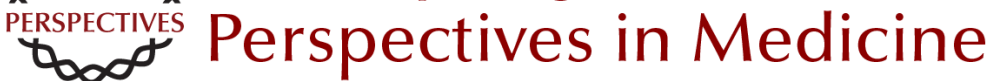

\section{Molecular Composition of Vestibular Hair Bundles}

Jocelyn F. Krey and Peter G. Barr-Gillespie

Cold Spring Harb Perspect Med 2019; doi: 10.1101/cshperspect.a033209 originally published online May 29, 2018

\section{Subject Collection Function and Dysfunction of the Cochlea}

Hidden Hearing Loss: A Disorder with Multiple Etiologies and Mechanisms

David C. Kohrman, Guoqiang Wan, Luis Cassinotti, et al.

Hair Cell Afferent Synapses: Function and Dysfunction

Stuart L. Johnson, Saaid Safieddine, Mirna Mustapha, et al.

Active Biomechanics of Sensory Hair Bundles Dolores Bozovic

The Tectorial Membrane: Mechanical Properties and Functions Jonathan B. Sellon, Roozbeh Ghaffari and Dennis M. Freeman

The Epidemiology of Deafness Abraham M. Sheffield and Richard J.H. Smith

Toward the Optical Cochlear Implant Tobias Dombrowski, Vladan Rankovic and Tobias Moser

Outer Hair Cells and Electromotility Jonathan Ashmore

Interactions between Macrophages and the Sensory Cells of the Inner Ear Mark E. Warchol
Development and Patterning of the Cochlea: From Convergent Extension to Planar Polarity Mireille Montcouquiol and Matthew W. Kelley

Hair-Bundle Links: Genetics as the Gateway to Function

Guy P. Richardson and Christine Petit

Aminoglycoside- and Cisplatin-Induced

Ototoxicity: Mechanisms and Otoprotective

Strategies

Corné J. Kros and Peter S. Steyger

Function and Dysfunction of TMC Channels in Inner Ear Hair Cells

David P. Corey, Nurunisa Akyuz and Jeffrey R. Holt

Cochlear Gene Therapy

Lawrence Lustig and Omar Akil

Age-Related Hearing Loss

Michael R. Bowl and Sally J. Dawson

Inner Ear Connexin Channels: Roles in

Development and Maintenance of Cochlear

Function

Fabio Mammano

A Functional Perspective on the Evolution of the Cochlea Christine Köppl and Geoffrey A. Manley

For additional articles in this collection, see http://perspectivesinmedicine.cshlp.org/cgi/collection/ 